\title{
Variabilidade na coordenação motora: uma abordagem centrada no delineamento gemelar
}

CDD. 20.ed. 152.385

616.042

\begin{tabular}{|c|c|}
\hline $\begin{array}{r}\text { Raquel Nichele de CHAVES* } \\
\text { Go TANI* } \\
\text { Michele Caroline de SOUZA* } \\
\text { Daniel SANTOS } \\
\text { J osé MAIA* }\end{array}$ & $\begin{array}{l}\text { *Faculdade de Des } \\
\text { porto, Universidade do } \\
\text { Porto - Portugal. } \\
\text { **Escola de Educa } \\
\text { ção Física e Esporte } \\
\text { Universidade de Sã } \\
\text { Paulo. }\end{array}$ \\
\hline
\end{tabular}

\begin{abstract}
Resumo
0 propósito deste estudo foi estimar a contribuição dos fatores genéticos e ambientais na variabilidade do desempenho interindividual na coordenação motora. A amostra foi constituída por 64 pares de gêmeos portugueses, com idades entre cinco e 14 anos. Avaliou-se o desempenho da coordenação através das quatro provas da bateria KTK: equilibrio à retaguarda (ER); saltos monopedais (SM); transposição lateral $(\mathrm{TL})$; saltos laterais (SL). Calculou-se o coeficiente de correlação intraclasse (t), sendo estimada a contribuição dos fatores genéticos $\left(\mathrm{a}^{2}\right)$, ambientais comuns $\left(\mathrm{c}^{2}\right)$ e únicos $\left(\mathrm{e}^{2}\right)$. Os resultados mostram valores de $t$ mais elevados entre gêmeos monozigóticos, sugerindo presença de fatores genéticos. Entretanto, estimativas de $a^{2}$ foram baixas, variando entre 15\% (TL) e 41\% (SM), enquanto para $c^{2}$ situaram-se entre 46\% (SL) e 58\% (TL), e para e entre 11\% (SM) e 28\% (TL). Conclui-se que fatores ambientais são responsáveis pela maior parcela de influência na variabilidade do desempenho na coordenação motora.
\end{abstract}

UNITERMOS: Coordenação Motora; Genética quantitativa; KTK.

\section{Introdução}

A expressão diferenciada de níveis de coordenação motora representa uma faceta de importância inquestionável no processo de aprendizagem de habilidades motoras, bem como no desenvolvimento motor de crianças e jovens. Crianças que apresentam movimentos precisos e equilibrados, e que sejam capazes de manifestar respostas rápidas e adequadas a cada situação, têm uma elevada probabilidade de sucesso no desempenho de suas tarefas diárias, sejam elas acadêmicas e/ou desportivas (HEMGREN \& Persson, 2009; Missiuna, Moll, King \& Law, 2006). Esse sucesso tende também a se repercutir, positivamente, em outros domínios como no seu relacionamento intra e interpessoal (ЕMCK, BOSSCHER, BeEK \& Doreleijers, 2009) e está associado de modo relevante ao contexto escolar e às oportunidades de aprendizagem motora orientada, potencializadas, sobretudo, nas aulas de Educação Física. De fato, é neste espaço temporal e letivo que partes significativas das aprendizagens e do desenvolvimento motor se realizam de forma estruturada, dirigidas para objetivos educativos bem definidos.

Ações motoras eficientes e respostas motoras rápidas emergem da interação precisa e econômica dos sistemas muscular, neural e sensorial (KIPHARD, 1976). O seu desenvolvimento ocorre em função da individualidade biológica de cada criança, do seu nível de maturação e de experiências motoras, aos quais se associa uma enorme variedade de fatores ambientais (BOUCHARD, MaLINA \& Pérusse, 1997; Laskowski, NewComer-Aney \& Smith, 2000; LeE, 1984; Malina, 2004). Em termos populacionais, no universo infanto-juvenil, é indiscutível a presença de elevada variabilidade interindividual nas mais variadas características, cuja expressão, por exemplo, na coordenação motora, é condicionada de modo aditivo e interativo, não só por fatores intrínsecos aos indivíduos, mas também pelas características dos seus contextos ambientais. A representação dessas diferenças tem sido expressa de modo quantitativo (VALDÍVIA, LARA, Espinoza, Pomahuacre, Ramos, Seabra, Garganta \& 
Maia, 2008; Vidal, Bustamante, Lopes, Seabra, Silva \& MAIA, 2008), não só pela distribuiçãao percentílica de cada um dos testes que marcam diferentes fatores da coordenação motora (MAIA, LOPES, BustaMANTE, Santos, Bacalhau, Silvi \& Seabra, 2007), mas também pela variância dos desempenhos em cada valor discreto de idade.

A existência de diferenças interindividuais sugere, necessariamente, uma análise da variância total dos resultados no desempenho motor, expressa nas mais diversas formas de avaliação da coordenação motora, bem como dos modos da sua partição. A estrutura conceitual, metodológica e analítica do paradigma da Genética Quantitativa é um bom candidato para abordar o problema do fracionamento e interpretação da variância total do desempenho coordenativo (MAIA, Loos, Beunen, Thomis, Vlietinck, Morais \& Lopes, 1999). Decorre daqui a noção central que o resultado obtido pela aplicação de qualquer teste que marque uma faceta da coordenação motora é um fenótipo quantitativo contínuo, de natureza complexa e multifatorial.

A informação disponível sobre a magnitude e os mecanismos da influência dos fatores genéticos na coordenação motora ainda é extremamente limitada (Malina, 2004). Francks, Fisher, Marlow, MacPhie, TAYLOR, Richardson e Stein (2003) analisaram relaçôes familiares entre medidas de habilidade motora manual e lateralidade, bem como o desempenho cognitivo numa amostra de 641 irmãos pertencentes a 265 famílias nucleares. Os resultados sugerem a presença de componente genético, responsável por
$23 \%$ e $42 \%$ da variância total na habilidade motora manual e na lateralidade, respectivamente. Evidências da contribuição genética também têm sido reportadas em análises de características motoras no primeiro ano de vida (Freedman \& Keller, 1963; Saudino \& EATON, 1991; Wilson \& Harpring, 1972), na expressão diferenciada de padrões de movimento (GoYA, Amano, Hoshikawa \& Matsui, 1993; Livshits, 1988) e desordens da coordenação motora (MARTIN, PIEK \& HAY, 2006), bem como em pesquisas com modelo animal acerca dos efeitos de mutaçôes de genes candidatos em padrōes de coordenação (Lalonde, HaYzoun, Selimi, Mariani \& STRAZIELLE, 2003; LALONDE \& Qian, 2007; Saleh, Espinosa de los Monteros, De Arriba Zerba, Fontaine, Piaud, Djordjievic \& Baroukh, 2003). No entanto, não tem sido fácil referenciar estudos de Genética Quantitativa relativos à coordenação motora grossa de crianças e jovens, sem diagnóstico de necessidades motoras especiais.

É consistentemente reportado na literatura que os delineamentos gemelares representam uma forma adequada de ajudar a esclarecer o quanto da variância total, em um qualquer fenótipo quantitativo complexo, é devido à presença de fatores genéticos e/ou ambientais, atuando de modo aditivo ou em interação e covariação (Dilalla, 2002; Neale \& Cardon, 1992). Daqui que o propósito deste estudo seja o de estimar a contribuição dos fatores genéticos e ambientais na variabilidade do desempenho interindividual nos testes de coordenação motora, em uma amostra de gêmeos.

\section{Procedimentos metodológicos}

\section{Amostra}

O presente estudo é de natureza transversal sendo constituído por uma amostra de 37 pares de gêmeos monozigóticos (MZ) e 27 pares de gêmeos dizigóticos (DZ), com idades compreendidas entre os cinco e os 14 anos (TABELA 1), provenientes do Concelho da Maia e Distrito de Viseu, norte e centro de Portugal, respectivamente. A amostra foi definida com base no voluntariado das famílias (trata-se, pois, de uma amostra de conveniência), e obtida em encontros de gêmeos viabilizados pelas respectivas Prefeituras Municipais. Todas as avaliações (coordenação motora e colheita de sangue) ocorreram no mesmo dia e foram realizadas em salas equipadas de ginásios municipais, somente após assinatura do consentimento livre e informado dos pais das crianças e adolescentes envolvidos. Este projeto teve a aprovação do Conselho Científico da Faculdade de Desporto da Universidade do Porto.

TABELA 1 - Distribuição amostral e intervalo etário dos pares de gêmeos por zigotia e sexo.

\begin{tabular}{clcc}
\hline \multicolumn{1}{c}{ Zigotia } & Pares $(\mathbf{n})$ & Idade $\mathbf{M} \pm \mathbf{d p}$ \\
\hline \multirow{2}{*}{$\mathrm{MZ}$} & irmão-irmão & 27 & $8 \pm 2$ \\
& irmã-irmã & 10 & $9 \pm 3$ \\
\hline \multirow{4}{*}{$\mathrm{DZ}$} & irmão-irmão & 14 & $10 \pm 2$ \\
& irmã-irmã & 5 & $8 \pm 3$ \\
& irmão-irmã & 8 & $12 \pm 2$ \\
\hline
\end{tabular}

MZ: monozigóticos; DZ: dizigóticos; M: média; dp: desvio-padrão. 


\section{Coordenação motora}

O desempenho da coordenação motora foi avaliado em cada um dos encontros de gêmeos por uma equipe previamente treinada, sendo realizada em estações referentes a cada um dos testes. A bateria de testes utilizada foi a Körperkoordinationtest für Kinder (KTK) desenvolvida pelos pesquisadores alemães Schiling e Kiphard (SCHILLING, 1974), cuja versão em português pode ser obtida, por exemplo, em GOMES (1996). A bateria é constituída por quatro testes que caracterizam as várias facetas da coordenação grossa e do domínio corporal: 1) equilíbrio à retaguarda (ER); 2) saltos monopedais (SM); transposição lateral (TL); e saltos laterais (SL). A partir do somatório de todas as provas e com base em tabelas construídas para o efeito pelos autores alemães, é possível obter um valor único, designado de quociente motor (QM).

\section{Determinação da zigotia}

A análise ao DNA dos membros de cada par implicou obtenção de uma amostra muito reduzida de sangue, uma única gota de calibre médio que foi vertida numa pequena tira de papel utilizada em pesquisa Forense para colheita de vestígios biológicos, sendo realizada por um profissional de saúde experiente. $\mathrm{O}$ método aplicado para obter o DNA fez uso de resina Chelex (LAREU, Phillips, Carracedo, Lincoln, Syndercombe Court \& Thomson, 1994). Em todas as amostras de DNA, a análise de 17 STRs autossômicos (CSF1PO, D2S1338, D3S1358, D5S818, D7S820, D8S1179, D13S317, D16S539, C18S51, D19S433, D21S11, FGA, PD, PE, TH01, TPO e VWA) e o "locus" da Amelogenina (determinação do sexo) foram efetuados por amplificação por PCR, utilizando os kits comerciais Powerplex 16 System (Promega Corporation) e Identifiler (AB Applied Biosystems), de acordo com as instruções dos fabricantes. A genotipagem foi efetuada em aparelhos ABI 310 Genetic Analyser (AB Applied Biosystems), seguindo as instruções do fabricante, por determinação do tamanho dos fragmentos de DNA e comparação com escalas alélicas fornecidas com os kits comerciais.

Nos casos em que os indivíduos apresentavam perfis genéticos de STRs idênticos, foi efetuado o cálculo de probabilidade de monozigotia, que segue, fundamentalmente, a metodologia de EsSEN-MøLLER (1939).

\section{Procedimentos estatísticos}

Inicialmente foi efetuada a análise exploratória e descritiva dos dados para a verificação de possíveis erros da informação ou presença de valores extremos. Face à reduzida dimensão amostral, optouse pela remoção dos efeitos de covariáveis (sexo, idade e respectivas interações), em um modelo de regressão múltipla, para lidar, exclusivamente, com gêmeos $\mathrm{MZ}$ e DZ, aumentando assim a potência das análises estatísticas e generalização dos resultados (Bouchard, Malina \& Pérusse, 1997). Os resíduos de regressão múltipla correspondem, desse modo, a fenótipos "mais precisos" de cada um dos testes da bateria KTK.

A variância intrapar em cada zigotia foi utilizada para calcular o coeficiente de correlação intraclasse (t). Esse coeficiente é uma estatística que expressa o grau de homogeneidade de uma classe de valores. Optou-se pela aplicação das fórmulas sugeridas por SNEDECOR e CoCHRAN (1989), onde os valores de $t$ são obtidos a partir da análise de variância (ANO$\mathrm{VA}$ ), contrastando-se médias de quadrados intrapar (MSW) relativamente à média dos quadrados entre pares (MSB). É previsto que os gêmeos MZ apresentem valores mais homogêneos do que os DZ, isto é, que os valores de correlação intraclasse sejam superiores aos dos DZ (Maia, Garganta, Seabra \& LOPES, 2004).

Os fatores genéticos e ambientais, bem como a sua interação e covariação são agentes responsáveis pela possível explicação da variabilidade dos valores de coordenação motora, se bem que seja assumido ausência de efeitos de interação e/ou covariação entre genes e ambiente no modelo aditivo, bem como efeitos de epistasia (Bouchard, Malina \& Pérusse, 1997; FALCONER \& MaCKaY, 1996). Em termos populacionais, a variância fenotípica total de qualquer fenótipo contínuo pode ser fracionada em dois tipos de variância: genética $(G)$ e do meio ambiente (do inglês "environmental") (E). A partir dos valores de correlação intraclasse $(\mathrm{t})$ obtidos dos resultados do desempenho dos gêmeos MZ e DZ é possível estimar a contribuição dos fatores genéticos e ambientais. Esses fatores, genético e ambiental, devem ser entendidos, o primeiro no sentido Mendeliano do termo, e o segundo do mesmo modo como os psicometristas entendem o significado de um fator extraído da análise fatorial. São, pois, variáveis latentes, não diretamente observadas. As frações da variância total correspondem ao contributo aditivo de fatores genéticos $\left(\mathrm{a}^{2}\right)$, que indica a quantidade de variância total observada correspondente à variância genética, ou a diferenças genéticas interindividuais. Podem-se estimar, também, as contribuiçôes dos fatores do ambiente comum $\left(\mathrm{c}^{2}\right)$, partilhados no 
seio familiar, e dos fatores únicos $\left(\mathrm{e}^{2}\right)$, específicos de cada um dos membros do par a que se adiciona variância residual e erros de medição, de acordo com fórmulas bem conhecidas (Dilalla, 2002; Neale \& Cardon, 1992):

$$
\mathrm{a}^{2}=2\left(\mathrm{t}_{\mathrm{MZ}}-\mathrm{t}_{\mathrm{DZ}}\right) ; \quad \mathrm{c}^{2}=2 \mathrm{t}_{\mathrm{DZ}}-\mathrm{t}_{\mathrm{MZ}} ; \quad \mathrm{e}^{2}=1-\mathrm{t}_{\mathrm{MZ}}
$$

\section{Resultados}

Os resultados dos ajustamentos para as covariáveis consideradas no estudo, bem como os valores de $\mathrm{R}^{2}$ ajustado, estão descritos na TABELA 2. Nos modelos de regressão múltipla (método "stepwise") foram obtidas diferentes soluçôes para cada um dos testes da bateria KTK, assim como para o QM. A contribuição de efeitos aditivos e multiplicativos na variância total do desempenho da coordenação situa-se entre os 15 (QM) e os 61\% (SM). Isso significa, por exemplo, que nas provas TL e SM a capacidade explicativa das covariáveis sexo, idade ${ }^{2}$, idade* sexo e idade ${ }^{*}$ sexo é de 41 e $61 \%$, respectivamente. Na prova SL, 36\% da variância no desempenho são explicados unicamente pela idade.

A similaridade intrapar nos gêmeos $\mathrm{MZe} \mathrm{DZ}$ ébem destacada nos diagramas de dispersão (FIGURA 1), sendo de realçar a menor variabilidade nos gêmeos MZ.

Os valores t e respectivos intervalos de confiança $\left(\mathrm{IC}_{95 \%}\right)$ dos resíduos de regressão, referentes ao desempenho nas quatro provas do KTK e ao QM, em que $\mathrm{t}_{\mathrm{MZ}}$ e $\mathrm{t}_{\mathrm{DZ}}$ correspondem a correlaçôes intraclasse entre pares de gêmeos $\mathrm{MZ}$ e DZ, respectivamente. Considerando tais fórmulas e com base nos valores de coeficiente de correlação intraclasse dos gêmeos $\mathrm{MZ}$ e $\mathrm{DZ}$, foi estimada a contribuição dos fatores genéticos $\left(\mathrm{a}^{2}\right)$, do ambiente comum $\left(\mathrm{c}^{2}\right)$ e únicos $\left(\mathrm{e}^{2}\right)$. Os cálculos foram realizados no "software" STATA 10.

estão descritos na TABELA 3. Nos gêmeos MZ, os valores de $t$ situam-se entre 0,73 (TL) e 0,89 (SM). Nos gêmeos DZ, essa variação está entre 0,61 (SL) e 0,69 (SM). Portanto, os gêmeos MZ tendem a apresentar uma variância intrapar mais reduzida, isto é, valores superiores de t.

As estimativas de efeitos genéticos $\left(\mathrm{a}^{2}\right)$, ambientais comuns $\left(\mathrm{c}^{2}\right)$ e únicos $\left(\mathrm{e}^{2}\right)$ estão descritas na TABELA 4. A contribuição genética é baixa, entre 15\% (TL) e $41 \%(\mathrm{SM})$ da variância fenotípica total. Para o QM o valor é de 39\%. O ambiente comum, ou seja, aquele partilhado no seio familiar, apresenta a maior influência no desempenho da coordenação motora de todas as provas, entre $46 \%$ (SL) e $58 \%$ (TL), bem como no QM (48\%). A amplitude dos resultados dos efeitos ambientais únicos $\left(\mathrm{e}^{2}\right)$ situa-se entre $11 \%$ (SM) e $28 \%$ (TL). Desse modo, verifica-se maior influência dos fatores ambientais relativamente aos fatores genéticos na variabilidade do desempenho da coordenação motora.

TABELA 2 - Efeitos das covariáveis na variância do desempenho em cada prova da bateria de testes KTK, bem como do quociente motor.

\begin{tabular}{|c|c|c|c|}
\hline \multirow{6}{*}{$\begin{array}{l}\mathrm{R}^{2}=\text { variância explicada } \\
\text { pelas covariáveis; } \\
\mathrm{ER}=\text { equilíbrio à } \\
\text { retaguarda; } \\
\mathrm{SL}=\text { salto lateral; } \\
\mathrm{TL}=\text { transposição } \\
\text { lateral; } \\
\mathrm{SM}=\text { salto monopedal; } \\
\mathrm{OM}=\text { a }\end{array}$} & Fenótipos & $\mathbf{R}^{2}$ ajustado (\%) & Covariáveis \\
\hline & ER & $31 \%$ & Idade, sexo e idade ${ }^{2}$ \\
\hline & SL & $36 \%$ & Idade \\
\hline & TL & $41 \%$ & Sexo, idade ${ }^{2}$, idade*sexo, idade ${ }^{2 *}$ sexo \\
\hline & SM & $61 \%$ & Sexo, idade ${ }^{2}$, idade $^{*}$ sexo, idade ${ }^{2 *} \operatorname{sexo}$ \\
\hline & QM & $15 \%$ & Idade e sexo \\
\hline
\end{tabular}


ER

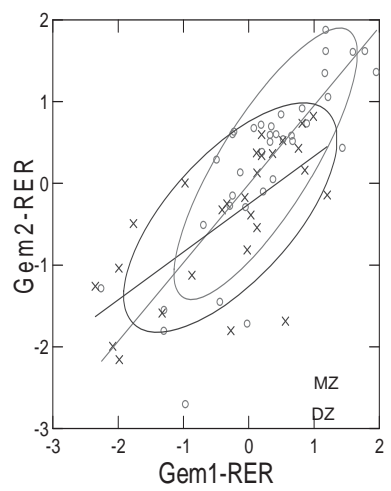

SL

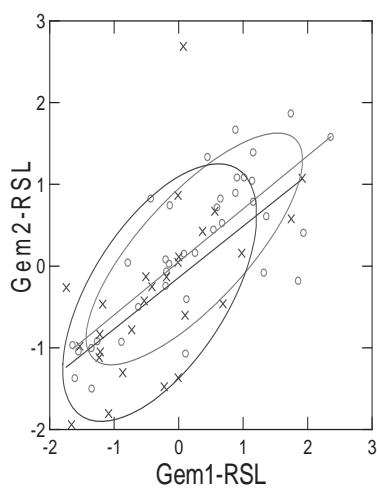

$\mathrm{TL}$

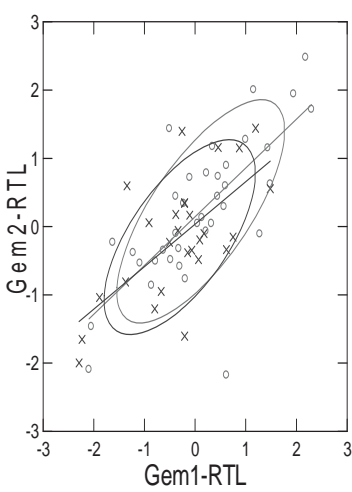

$E R$ = equilíbrio à retaguarda;

$\mathrm{SL}=$ salto lateral

$\mathrm{TL}=\operatorname{transposição~}$ lateral;

$\mathrm{SM}=$ salto monopedal; $\mathrm{QM}=$ quociente motor.
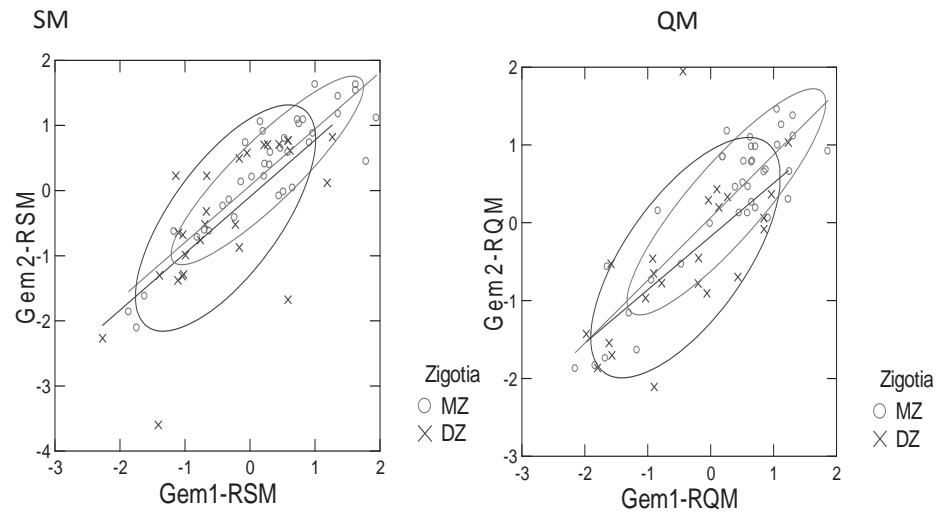

FIGURA 1 - Diagramas de dispersão intrapar e respectivas elipses do desempenho dos gêmeos MZ e DZ nas provas da bateria de testes KTK e do QM (os eixos representam resíduos de regressão).

TABELA 3 - Valores de correlação intraclasse eerro-padrão ( $t \pm e p$ ) do desempenho nas provas do KTKequociente motor, bem como intervalos de confiança ( $\left.\mathrm{IC}_{95} \%\right)$.

\begin{tabular}{lccc}
\hline & Fenótipos & $\mathbf{t} \pm \mathbf{e p}$ & IC $_{\mathbf{9 5}} \mathbf{\%}$ \\
\hline \multirow{2}{*}{ Equilíbrio à Retaguarda } & MZ & $0,81 \pm 0,06$ & 0,$69 ; 0,92$ \\
& DZ & $0,68 \pm 0,11$ & 0,$47 ; 0,88$ \\
\hline \multirow{2}{*}{ Salto Lateral } & MZ & $0,76 \pm 0,07$ & 0,$63 ; 0,89$ \\
\hline \multirow{2}{*}{ Transposição Lateral } & DZ & $0,61 \pm 0,12$ & 0,$37 ; 0,85$ \\
\hline \multirow{2}{*}{ Salto Monopedal } & MZ & $0,73 \pm 0,08$ & 0,$57 ; 0,88$ \\
& DZ & $0,65 \pm 0,11$ & 0,$43 ; 0,87$ \\
\hline \multirow{2}{*}{ Quociente Motor } & MZ & $0,89 \pm 0,03$ & 0,$83 ; 0,96$ \\
& DZ & $0,69 \pm 0,10$ & 0,$49 ; 0,89$ \\
\hline
\end{tabular}

$\mathrm{MZ}=$ monozigóticos;

TABELA 4 - Estimativas de efeitos genéticos $\left(\mathrm{a}^{2}\right)$, ambientais comuns $\left(\mathrm{c}^{2}\right)$ e únicos $\left(\mathrm{e}^{2}\right)$.

\begin{tabular}{lccc}
\hline Fenótipo & $\mathbf{a}^{\mathbf{2}}$ & $\mathbf{c}^{\mathbf{2}}$ & $\mathbf{e}^{\mathbf{2}}$ \\
\hline Equilíbrio à Retaguarda & 0,26 & 0,55 & 0,19 \\
Salto Lateral & 0,30 & 0,46 & 0,24 \\
Transposição Lateral & 0,15 & 0,58 & 0,28 \\
Salto Monopedal & 0,41 & 0,48 & 0,11 \\
Quociente Motor & 0,39 & 0,48 & 0,32 \\
\hline
\end{tabular}

Rev. bras. Educ. Fís. Esporte, São Paulo, v.26, n.2, p.301-11, abr./jun. 2012 • 305 


\section{Discussão}

$\mathrm{Na}$ introdução deste trabalho ressaltamos que um dos desafios da Educação Física no âmbito escolar está na construção de um contexto de aprendizagem diferenciado para o desenvolvimento da coordenação motora de todas as crianças. Não obstante a exigência de uma didática específica a cada grupo etário, o professor de Educação Física é confrontado com a presença de elevada variabilidade interindividual dos níveis de coordenação entre crianças ou adolescentes de mesma idade. Com base nos valores percentílicos do desempenho da coordenação motora, estudos prévios (MAIA et al., 2007; VAldívia et al., 2008; VidAL et al., 2008) salientam a forte variação nos valores em cada valor discreto de idade e em cada prova da Bateria de Testes KTK, bem expressos na amplitude de resultados situados entre os percentis 3 e 97 .

Tendo em conta os diferentes perfis configuracionais que ilustram a enorme variabilidade interindividual expectável em determinada idade, espera-se que a ação educativa do professor considere a peculiaridade dos níveis de coordenação de cada indivíduo, propondo vivências motoras diferenciadas, com múltiplas oportunidades de prática, modos de instrução e de estímulo. Propostas de experiências motoras significativas são favorecidas quando há conhecimento prévio em dois domínios: 1) sobre a variância dos níveis de coordenação motora em cada valor discreto de idade, bem descrita pelos estudos supracitados (MAIA et al., 2007; VALDÍVIA et al., 2008; VIDAL et al., 2008); 2) quanto à magnitude de influência dos fatores envolvidos, nomeadamente genéticos e ambientais. Daqui que o principal propósito deste estudo tenha sido estimar a contribuição dos fatores genéticos e ambientais na variabilidade interindividual dos níves de coordenação motora.

Em uma primeira análise, com o cálculo dos coeficientes de correlação intraclasse $(t)$, pode-se interpretar a variância intrapar em cada zigotia e identificar a possível presença de efeitos genéticos aditivos. Os resultados mostram valores de $\mathrm{t}$ mais elevados nos gêmeos MZ comparativamente aos gêmeos $\mathrm{DZ}$, mostrando que os níveis de coordenação motora entre cada par de gêmeos $\mathrm{MZ}$ são mais homogêneos e concordantes. Não é do nosso conhecimento qualquer relato prévio referente à presença e magnitude de efeitos genéticos em distintos fenótipos marcadores da coordenação motora grossa. No entanto, estudos gemelares têm reportado algumas evidências de influências genéticas em características motoras de crianças no primeiro ano de vida (FreEdMAN \& KELLER, 1963; SAUdino \& EATON, 1991; WiLSON \& Harpring, 1972). Em geral, os três estudos avaliaram aspectos do desenvolvimento motor de bebês gêmeos, por meio de um índice de desenvolvimento psicomotor obtido com a Escala de Bayley de Desenvolvimento Infantil. FreEdMan e KeLLER (1963) avaliaram 20 pares de bebês gêmeos $\mathrm{MZ}$ e DZ, mensalmente, durante o primeiro ano de vida, verificando que entre gêmeos $\mathrm{DZ}$ há menor similaridade intrapar do que entre gêmeos MZ. O estudo de WiLSON e HARPRING (1972) envolveu 261 pares de bebês MZ e DZ, acompanhando o desenvolvimento motor com avaliações trimestrais, dos três aos 18 meses de idade, e uma última avaliação quando os bebês completaram 24 meses. Os autores reportam coeficientes de correlação intraclasse específicos a cada idade mostrando, também, maior concordância intrapar entre os gêmeos $\mathrm{MZ}$ na maioria das habilidades motoras avaliadas. $\mathrm{O}$ mesmo é apresentado por SAUDINO e EATON (1991) em uma amostra de 60 pares de bebês gêmeos com idade cronológica média de 30,7 semanas. Essas evidências corroboram os resultados do presente estudo, sugerindo a dependência de influências genéticas nas características motoras avaliadas.

Embora a magnitude das correlaçóes obtidas neste estudo seja maior para os gêmeos $\mathrm{MZ}$, os valores de correlação entre gêmeos DZ, nos quatro testes, apresentam similaridade moderada, entre 0,61 e 0,69, semelhantes aos reportados por WILSON e HARPRING (1972), com valores de correlação entre 0,41- 0,77, respectivamente. Os gêmeos $\mathrm{DZ}$ partilham, entre si, metade dos genes idênticos por descendência, sendo de esperar alguma similitude no seu desempenho da coordenação. Entretanto, quando a similaridade em gêmeos DZ é maior do que seria predito pelo modelo clássico de estudos gemelares, fica evidente a presença de influências não-genéticas na expressão fenotípica em causa (SAUDINO \& EATON, 1991), tais como o ambiente familiar, escolar e a vizinhança, as quais são comuns a cada membro do par.

No presente estudo, a influência dos fatores ambientais partilhados pelos pares de gêmeos situa-se entre $46 \%$ e $58 \%$ da variância total do desempenho nas provas da bateria de testes KTK, enquanto para os fatores ambientais únicos - não partilhados, essa influência situa-se entre 11 e 32\%. Não é comum em estudos prévios sobre traços da coordenação motora o cálculo de estimativas da fração de influência 
devido a fatores genéticos, ambientais comuns e do ambiente único de modo isolado. A informação centra-se, sobretudo, na presença de efeitos genéticos e ambientais, não estimando a contribuição específica do ambiente partilhado. Além disso, é do nosso conhecimento um único estudo que utiliza um modelo de "path analysis" (análise de trajetórias) designado ACE ( $\mathrm{A}$ = efeitos genéticos aditivos; $\mathrm{C}=$ ambiente partilhado e $\mathrm{E}=$ ambiente único e aleatório) para estimar a magnitude desses componentes nos níveis da coordenação motora grossa e geral, mas somente em distúrbios da coordenação motora. Martin, Piek e Hay (2006) investigaram a etiologia partilhada de desordens como o transtorno do déficit de atenção e hiperatividade (TDAH) e a desordem de coordenação motora, em uma amostra de 1285 pares de gêmeos $\mathrm{MZ}$ e DZ, com idades entre os cinco e os 16 anos. No que concerne à coordenação motora, foram avaliados quatro traços: controle durante o movimento, coordenação motora fina, coordenação motora grossa e coordenação motora global. Os resultados revelam uma forte componente genética aditiva, com estimativas que variam entre $64 \%$ e $85 \%$. A influência do ambiente partilhado corresponde a $20 \%$ em todos os traços avaliados, exceto na coordenação motora global. Os efeitos do ambiente único situam-se entre 7\% e 16\%. É provável que esses resultados estejam condicionados às especificidades da própria amostra. Aliás, é comum na literatura, em outros domínios de interesse da investigação (ver por exemplo Chaves, Souza, Santos, Garganta, Seabra \& Maia, 2010), encontrar valores distintos nas estimativas dos fatores genéticos e ambientais (comuns e únicos) que refletem disparidades em termos de dimensões amostrais, qualidade de fenótipo e sua medição, métodos do seu refinamento (por ajustamento, ou não, a distintas covariáveis), uso de procedimentos estatísticos diversos e, bem entendido, aspectos idiossincráticos das amostras.

Outros estudos têm centrado seus esforços na compreensão da influência genética em outros aspectos do desenvolvimento motor. GOETGHEBUER, Ota, Kebbeh, John, Jackson-Sillah, Vekemans e MARCHANT (2003) avaliaram a contribuição de fatores genéticos nos marcos iniciais de desenvolvimento motor em uma amostra de 22 pares de gêmeos $M Z$ e 62 pares de gêmeos DZ, provenientes de Gâmbia, oeste do continente africano. $\mathrm{O}$ desenvolvimento de oito marcos motores foi avaliado e estimativas de heritabilidade $\left(h^{2}\right)$ foram calculadas. Quatro marcos motores, nomeadamente, engatinhar, sentar, segurar algum objeto e caminhar com apoio apresentam contribuição genética de 93\%, 94\%, $72 \%$ e $90 \%$, respectivamente. Em um estudo com 66 famílias, LivisHits (1988) apresenta estimativas entre 0 e 0,42 , para o movimento "virar" e a caminhada, respectivamente. Convém ressaltar que as estimativas elevadas refletem não só a contribuição genética, mas também os efeitos do ambiente partilhado entre os pares de gêmeos, pois o modelo estatístico aplicado não permite estimar para esses efeitos separadamente.

Estudos referentes à contribuição genética na determinação de habilidades motoras também têm sido previamente reportados. Williams e Gross (1980) estudaram a extensão da contribuição genética para a aprendizagem e performance de uma habilidade motora grossa. Com base nos coeficientes de correlação intraclasse, calcularam a variância ambiental sistemática e não-sistemática, bem como a variância fenotípica total (herdada). Os resultados indicam efeitos significativos genéticos nos perfis de aprendizagem, entretanto com forte influência de efeitos ambientais sistemáticos na performance e aprendizado. Fox, Hershberger e Bouchard (1996) sugerem que a performance motora reflete influência genética, no entanto que a similaridade ambiental pode reduzir as diferenças entre gêmeos e revelar estabilidade e aumento da $\mathrm{h}^{2}$. Os padróes de correlação encontrados também indicam a presença de efeitos não aditivos.

Não obstante as evidências significativas sobre os efeitos genéticos em diferentes traços da coordenação motora, é ainda limitado o recurso à Genética Molecular. Francks, Fisher, MacPhie, Richardson, Marlon, Stein e Monaco (2002), ao estudar a habilidade motora manual em 195 pares de irmãos, encontraram evidências de "linkage", i.e., identificação de uma região cromossômica que possa conter genes candidatos, no cromossomo 2p12-q11. Em uma nova amostra de 105 pares de irmãos, Francks, Delisi, Fisher, Laval, Rue, Stein e Monaco (2003) confirmaram essa evidência, sugerindo que há ao menos um polimorfismo localizado no cromossomo $2 \mathrm{p} 12-\mathrm{q} 11$ que influencia a determinação da lateralidade.

O presente estudo apresenta algumas limitações que importa salientar. A primeira refere-se à reduzida dimensão amostral, não obstante ser relativamente difícil obter amostras mais vastas em estudos desta natureza, sobretudo em avaliações relativamente morosas da coordenação motora. Por exemplo, o número de pares de gêmeos $\mathrm{MZ}$ e DZ do estudo de Missitzi, Geladas e Kuissouras (2004) sobre a heritabilidade na coordenação neuromuscular foi 
de 10 em cada zigotia. É possível que ao longo da idade, aspectos distintos do desenvolvimento da coordenação sejam afetados por fatores genéticos em magnitudes distintas. O recurso aos resíduos da regressão múltipla permite, por um lado, corrigir os fenótipos da coordenação para efeitos da idade e sexo. Por outro, mostra que a idade associada ao sexo, de modo ativo e/ou multiplicativo, explica uma parte importante da coordenação motora. A segunda limitação dos resultados está associada à ausência de informação sobre experiência prévia de prática desportiva sistematizada, a qual pode, necessariamente, associar-se à covariação positiva com diferentes testes de coordenação motora. E, finalmente, a terceira limitação reside na circunstância dos resultados do desempenho nas provas da bateria de testes KTK não terem sido ajustados, também, para eventuais efeitos da idade gestacional. Esse é um importante preditor do surgimento marcos motores e pode apresentar efeitos aditivos ou multiplicativos na variância do desempenho da coordenação (PETER, VAINDER \& LIVSHITS, 1999).

Contudo, este estudo apresenta alguns pontos fortes que precisam ser destacados. Em primeiro lugar, o método direto de identificação da zigotia, a partir do DNA de cada elemento da amostra. Em segundo lugar, o fato de não existir nenhum estudo gemelar centrado na coordenação motora avaliada com a bateria de testes KTK. E por fim, a determinação das estimativas de $\mathrm{h}^{2}$, sugerindo a fração de contribuição dos fatores envolvidos na expressão do desempenho da coordenação, haja vista que estudos anteriores não reportam a magnitude dos efeitos genéticos e ambientais.
Importa ressaltar que os valores reportados no presente estudo estão condicionados ao modo de avaliação da coordenação motora global pela Bateria de Testes KTK. Os resultados referem-se, portanto, aos quatro testes marcadores do desempenho coordenativo: 1) equilíbrio dinâmico; 2) força dinâmica e coordenação dos membros inferiores; 3) lateralidade e velocidade em saltos alternados; e 4) lateralidade e estruturação espaço-temporal. É provável que o recurso a outra Bateria de Testes, com uma estrutura diferenciada em termos de marcação de aspectos da coordenação motora, produzisse estimativas distintas. Daqui a nossa sugestão, em estudos futuros, dentro dos limites de tempo, financiamento, espaços, materiais, equipes e disponibilidade dos gêmeos avaliados, recorrer a distintas formas de avaliação da coordenação motora para ampliar a extensão do conhecimento acerca da influência dos fatores genéticos e ambientais.

Em conclusão, os resultados do presente estudo sugerem a presença de influência genética de baixa magnitude na variabilidade interindividual do desempenho na coordenação motora. Desse modo, os efeitos ambientais, partilhados e do ambiente único, assumem a maior importância na explicação das diferenças interindividuais do desempenho nas quatro provas da bateria de testes KTK. Portanto, é no ambiente familiar e escolar que se encontra o desafio de criar condiçóes e situaçóes para que as crianças e os adolescentes possam vivenciar experiências motoras diversas. Além disso, ressaltase daqui a necessidade de tornar tais experiências significativas, ou seja, coerentes ao contexto físico e social no qual a criança ou adolescente atua.

\title{
Notas
}

1. Raquel Nichele de Chaves e Michele Caroline de Souza são bolsitas CAPES (Doutorado no Exterior), Ministério da Educação.

\begin{abstract}
Variability in motor coordination: an approach based on the twin design

The purpose of this study was to estimate the contribution of environmental and genetic factors on the variability of interindividual performance in motor coordination. Sample comprised 64 twin pairs, aged 5 to 14 years old, from Portugal. Motor coordination was evaluated by KTK battery: backward balance (ER); hopping on one leg (SM); shifting platforms (TL); jumping sideways (SL). Intraclass correlation coefficient
\end{abstract}


$(t)$ was calculated. Contributions of genetic $\left(a^{2}\right)$, shared $\left(c^{2}\right)$ and unique environmental $\left(e^{2}\right)$ factors were estimated. Our results showed t values higher in monozygotic twins, suggesting the presence of genetic factors. Nevertheless, $a^{2}$ estimates were low, ranging from 15\% (TL) to $41 \%(\mathrm{SM})$, while to common environment $\left(\mathrm{C}^{2}\right)$ ranged from $46 \%(\mathrm{SL})$ to $58 \%$ (TL), and unique effects ranged from $11 \%(\mathrm{SM})$ to $28 \%$ (TL). These results suggest that environmental factors are responsible for the greatest part of influence on variability of interindividual performance in motor coordination tests.

UNITERMS: Motor coordination; Quantitative genetic; KTK.

\section{Resumen}

Variabilidad en la coordinación motora: un enfoque centrado en el delineamiento gemelar

El objetivo del presente estudio fue estimar la contribución de los factores genéticos y ambientales en la vEl objetivo del presente estudio fue estimar la contribución de los factores genéticos y ambientales en la variabilidad del desempeño interindividual en la coordinación motora. La muestra fue constituida por 64 pares de gemelos portugueses, con edades entre 5 y 14 años. Se evaluó el desempeño de la coordinación a través de las cuatro pruebas de la batería KTK: equilibrio a la retaguardia (ER); saltos monopedales (SM); transposición lateral (TL); saltos laterales (SL). Se calculó el coeficiente intraclase (t), siendo estimada la contribución de los factores genéticos $\left(\mathrm{a}^{2}\right)$, ambientales comunes $\left(\mathrm{c}^{2}\right)$ y únicos $\left(\mathrm{e}^{2}\right)$. Los resultados muestran valores $t$ mas elevados entre gemelos monocigóticos, sugiriendo la presencia de factores genéticos. Por otro lado, estimaciones de $a^{2}$ fueron bajas, variando entre $15 \%(\mathrm{TL})$ y $41 \%(\mathrm{SM})$, mientras para $\mathrm{c}^{2}$ se sitúan entre $46 \%$ (SL) y $58 \%$ (TL), y para e entre $11 \%(\mathrm{SM})$ y $28 \%$ (TL). Se concluye que factores ambientales son responsables por la mayor porción de influencia de la variabilidad del desempeño en la coordinación motora.

Palabras clave: Coordinación motora; Genética cuantitativa; KTK.

\section{Referências}

BOUCHARD, C.; MALINA, R.M.; PÉRUSSE, L. Genetics of fitness and physical performance. Champaign: Human Kinetics, 1997.

CHAVES, R.N.; SOUZA, M.C.; SANTOS, D.; GARGANTA, R.; SEABRA, A.; MAIA, J. Agregação familiar nos níveis de atividade física: um resumo do estado da arte. Revista Brasileira de Atividade Física e Saúde, Londrina, v. 15, n. 1, p.65-9, 2010. DILALLA, L.F. Behavioral genetics principles: perspectives in development, personality, and psychopathology. Washington: American Psychological Association, 2002.

EMCK, C.; BOSSCHER, R.; BEEK, P.; DORELEIJERS, T. Gross motor performance and self-perceived motor competence in children with emotional, behavioural, and pervasive developmental disorders: a review. Developmental Medicine and Child Neurology, Oxford, v.51, n.7, p.501-17, 2009.

ESSEN-MOLLER, E. Die beweiskraft der ahnlichkeit im vaterschftznachweiss: theoretische brundlagen. Mitteilungen der Anthropologischen Gesellschaft, Wien, v.68, p.9-53, 1939.

FALCONER, D.S.; MacKAY, T.F.C. Introduction to quantitative genetics. Harlow: Longmans Green, 1996.

FOX, P.W.; HERSHBERGER, S.L.; BOUCHARD JUNIOR, T.J. Genetic and environmental contributions to the acquisition of a motor skill. Nature, London, v.384, n.6607, p.356-8, 1996.

FRANCKS, C.; DELISI, L.E.; FISHER, S.E.; LAVAL, S.H.; RUE, J.E.; STEIN, J.F., MONACO, A.P. Confirmatory evidence for linkage of relative hand skill to 2p12-q11. American Journal of Human Genetics, Baltimore, v.72, n.2, p.499-502, 2003. FRANCKS, C.; FISHER, S.E.; MacPHIE, I.L.; RICHARDSON, A.J.; MARLOW, A.J.; STEIN, J.F.; MONACO, A.P. A genomewide linkage screen for relative hand skill in sibling pairs. American Journal of Human Genetics, Baltimore, v.70, n.3, p.800-5, 2002. 
FRANCKS, C.; FISHER, S.E.; MARLOW, A.J.; MacPHIE, I.L.; TAYLOR, K.E.; RICHARDSON, A.J.; STEIN, J.F. Familial and genetic effects on motor coordination, laterality, and reading-related cognition. American Journal of Psychiatry, Arlington, v.160, n.11, p.1970-7, 2003.

FREEDMAN, D.G.; KELLER, B. Inheritance of behavior in infants. Science, New York, v.140, n.356, p.196-8, 1963. GOETGHEBUER, T.; OTA, M.O.; KEBBEH, B.; JOHN, M.; JACKSON-SILLAH, D.; VEKEMANS, J.; MARCHANT, A. Delay in motor development of twins in Africa: a prospective cohort study. Twin Research, Bowen Hills, v.6, n.4, p.279-84, 2003

GOMES, M.P.B.B. Coordenação motora, aptidão física e variáveis do envolvimento: estudo em crianças do $1 .^{\circ}$ ciclo de ensino de duas freguesias do Concelho de Matosinhos. 1996 Tese (Doutoramento) - Faculdade de Ciências do Desporto e de Educação Física da Universidade do Porto, Porto, 1996.

GOYA, T.; AMANO, Y.; HOSHIKAWA, T.; MATSUI, H. Longitudinal study on the variation and development of selected sports performance in twins: case study for one pair of female monozygous (MZ) and dizygous (DZ) twins. Sport Science, [S.l.], v.14, p.151-168, 1993.

HEMGREN, E.; PERSSON, K. Deficits in motor co-ordination and attention at 3 years of age predict motor deviations in 6.5-year-old children who needed neonatal intensive care. Child: Care, Health and Development, Oxford, v.35, n.1, p.120-9, 2009. KIPHARD, E.J. Insuficiencias del movimiento y de coordenación en la edad de la escuela primaria. Buenos Aires: Kapeluscz, 1976.

LALONDE, R.; HAYZOUN, K.; SELIMI, F.; MARIANI, J.; STRAZIELLE, C. Motor coordination in mice with hotfoot, Lurcher, and double mutations of the Grid2 gene encoding the delta-2 excitatory amino acid receptor. Physiology \& Behavior, Oxford, v.80, n.2-3, p.333-9, 2003.

LALONDE, R.; QIAN, S. Exploratory activity, motor coordination, and spatial learning in Mchr1 knockout mice. Behavioural Brain Research, Amsderdam, v.178, n.2, p.293-304, 2007.

LAREU, M.V.; PHILLIPS, C.P.; CARRACEDO, A.; LINCOLN, P.J.; SYNDERCOMBE COURT, D.; THOMSON, J.A. Investigation of the STR locus HUMTH01 using PCR and two electrophoresis formats: UK and Galician Caucasian population surveys and usefulness in paternity investigations. Forensic Science International, Limerick, v.66, n.1, p.41-52, 1994. LASKOWSKI, E.R.; NEWCOMER-ANEY, K.; SMITH, J. Proprioception. Physical Medicine and Rehabilitation Clinics of North America, Philadelphia, v.11, n.2, p.323-40, 2000.

LEE, W.A. Neuromotor synergies as a basis for coordinated intentional action. Journal of Motor Behavior, Washington, v.16, n.2, p.135-70, 1984.

LIVSHITS, G. Environmental and sibling resemblance components of variance and covariance in traits of early child development. Anthropologischer Anzeiger, Stuttgart, v.46, n.1, p.41-50, 1988.

MAIA, J.; LOOS, R.; BEUNEN, G.; THOMIS, M.; VLIETINCK, R.; MORAIS, F.P.; LOPES, V.P. Aspectos genéticos da prática desportiva: um estudo em gêmeos. Revista Paulista de Educação Física, São Paulo, v.13, n.2, p.160-76, 1999. MAIA, J.A.R.; GARGANTA, R.; SEABRA, A.; LOPES, V. Heterogeneidade nos níveis de actividade física de crianças dos 6 aos 12 anos de idade: um estudo em gémeos. Revista Portuguesa de Ciências do Desporto, Porto, v.4, n.1, p.39-50, 2004. MAIA, J.A.R.; LOPES, V.P.; BUSTAMANTE, A.; SANTOS, M.L.; BACALHAU, F.; SILVA, R.G.; SEABRA, A. Crescimento e desempenho motor de crianças e jovens açorianos: cartas de referência para o uso em educação física, desporto, pediatria e nutrição. Açores: DRD-RAA/FADEUP, 2007.

MALINA, R.M. Motor development during infancy and early childhood: overview and suggested directions for research. International Journal of Sport and Health Science, Beijing, v.2, p.50-66, 2004.

MARTIN, N.C.; PIEK, J.P.; HAY, D. DCD and ADHD: a genetic study of their shared aetiology. Human Movement Science, Amsterdam, v.25, n.1, p.110-24, 2006.

MISSITZI, J.; GELADAS, N.; KLISSOURAS, V. Heritability in neuromuscular coordination: implications for motor control strategies. Medicine and Science in Sports and Exercise, Madison, v.36, n.2, p.233-40, 2004.

MISSIUNA, C.; MOLL, S.; KING, G.; LAW, M. "Missed and misunderstood": children with coordination difficulties in the school system. International Journal of Special Education, Vancouver, v.21, n.1, 2006.

NEALE, M.C.; CARDON, L. Metholology for genetic studies of twin and families. Dordrecht: Kluwer Academic, 1992. PETER, I.; VAINDER, M.; LIVSHITS, G. Genetic analysis of motor milestones attainment in early childhood. Twin Research, Bowen Hills, v.2, n.1, p.1-9, 1999.

SALEH, M.-C.; ESPINOSA DE LOS MONTEROS, A.; DE ARRIBA ZERPA, G.A.; FONTAINE, I.; PIAUD, O.; DJORDJIJEVIC, D.; BAROUKH, N. Myelination and motor coordination are increased in transferrin transgenic mice. Journal of Neuroscience Research, New York, v.72, n.5, p.587-94, 2003. 
SAUDINO, K.J.; EATON, W.O. Infant temperament and genetics: an objective twin study of motor-activity level. Child

Development, Lafayette, v.62, n.5, p.1167-74, 1991.

SCHILLING, F. Körperkoordinationstest für Kinder, KTK von F. Schilling und E. Kiphard: manual. Weinheim: Beltz Test Gmbh, 1974.

SNEDECOR, G.W.; COCHRAN, W.G. Statistical methods. Ames: Iowa State University Press, 1989.

VALDÍVIA, A.B.; LARA, R.; ESPINOZA, C.B.; POMAHUACRE, S.Q.; RAMOS, G.R.; SEABRA, A.; GARGANTA, R.; MAIA, J. Prontitud coordinativa: perfiles multivariados en función de la edad, sexo y estatus socio-económico. Revista Portuguesa de Ciências do Desporto, Porto, v.8, n.1, p.34-46, 2008.

VIDAL, S.M.; BUSTAMANTE, A.; LOPES, V.P.; SEABRA, A.; SILVA, R.G.; MAIA, J.A. Construção de cartas centílicas da coordenação motora de crianças dos 6 aos 11 anos da Região Autónoma dos Açores, Portugal. Revista Portuguesa de Ciências do Desporto, Porto, v.9, n.1, p.24-35, 2008.

WILLIAMS, L.R.; GROSS, J.B. Heritability of motor skill. Acta Geneticae Medicae et Gemellologiae, Roma, v.29, n.2, p.127-36, 1980.

WILSON, R.S.; HARPRING, E.B. Mental and motor development in infant twins. Developmental Psychology, Richmond, v.7, n.3, p.277-87, 1972.

\section{Agradecimentos}

Trabalho financiado pela Fundação para a Ciência e Tecnologia (FCT) de Portugal com a referência PTDC/ DES/67569/2006.

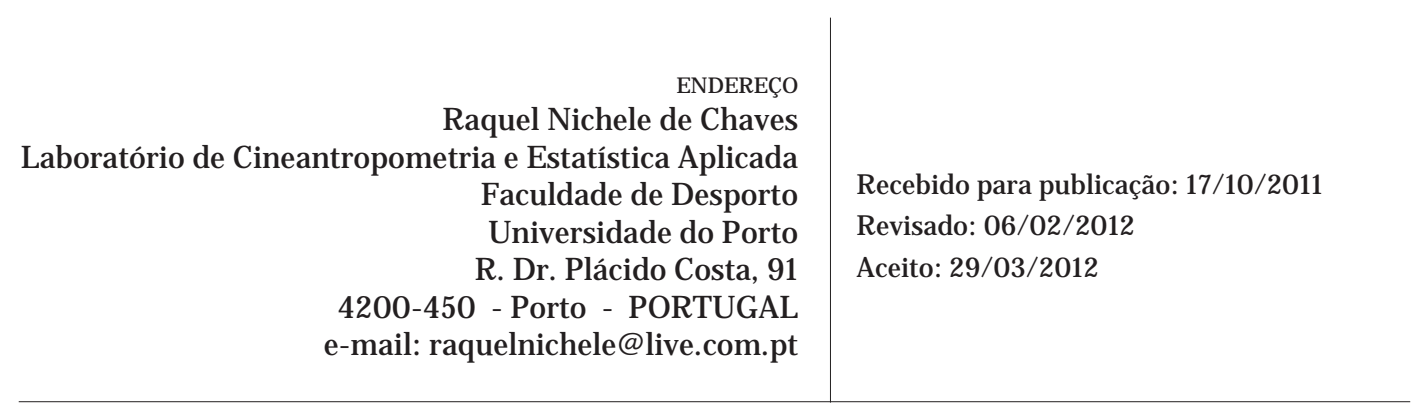

